\title{
Determination of Dynamic Viscosity in Samples of Blood Plasma and Hemoglobin Solution Using Nuclear Magnetic Resonance
}

\author{
Yulianela Mengana Torres ${ }^{1,}$ *, Manuel Arsenio Lores Guevara ${ }^{1}$, Juan Carlos García Naranjo ${ }^{1}$, \\ Beatriz Taimy Ricardo Ferro', Lidia Clara Suárez Beyries ${ }^{2}$, Inocente Clemente Rodríguez Reyes ${ }^{2}$, \\ María A. Marichal Feliu², Yomaidis Araujo Durán ${ }^{2}$ \\ ${ }^{1}$ Centro de Biofísica Médica, Universidad de Oriente, Patricio Lumumba S/N. Santiago de Cuba. Cuba \\ ${ }^{2}$ Hospital General "Dr. Juan Bruno Zayas Alfonso", Carretera del Caney S/N, Santiago de Cuba. Cuba
}

Email address:

yulianelatorres@gmail.com (Y. M. Torres), yulianela.mengana $a$ cbiomed.cu (Y. M. Torres)

${ }^{*}$ Corresponding author

To cite this article:

Yulianela Mengana Torres, Manuel Arsenio Lores Guevara, Juan Carlos García Naranjo, Beatriz Taimy Ricardo Ferro, Lidia Clara Suárez Beyrio, Inocente Clemente Rodríguez Reyes, María Altagracia Marichal Feliu, Yomaidis Araujo Durán. Determination of Dynamic Viscosity in Samples of Blood Plasma and Hemoglobin Solution Using Nuclear Magnetic Resonance. International Journal of Biochemistry, Biophysics \& Molecular Biology. Vol. 4, No. 2, 2019, pp. 25-30. doi: 10.11648/j.ijbbmb.20190402.12

Received: December 11, 2018; Accepted: January 14, 2019; Published: January 10, 2020

\begin{abstract}
The viscosity evaluation in Plasma is extremely useful in the clinical evaluation of different diseases. A procedure is presented, based on Protonic Magnetic Resonance, for the evaluation of the dynamic viscosity in Blood Plasma and in hemoglobin solution from the determination of the transverse relaxation time $\left(T_{2}\right)$. To experimentally determine the $T_{2}$ value, the impulse series Carr-Purcell-Meiboon-Gill was used in a MARAN DRX console (OXFORD INSTRUMENTS) and a homogeneous magnetic system $\left(\mathrm{B}_{0}=0.095 \mathrm{~T}\right)$. Values were obtained for the viscosity of the blood plasma and hemoglobin of $1.68 \pm 0.12 \mathrm{mPas}$ and $12.78 \pm 3.55 \mathrm{mPas}$ respectively, which agreed with the determined, in the same samples, using an Ostwald viscometer (1, $45 \pm 0.06 \mathrm{mPas}$ for the plasma and $12.82 \pm 3.35 \mathrm{mPas}$ for the dissolution of hemoglobin). The dynamic viscosity of the blood plasma was determined in 236 patients with Multiple Myeloma $(2.19 \pm 0.58 \mathrm{mPas}), 142$ with Drepanocytic Anemia (2.20 $\pm 0.79 \mathrm{mPas})$ showing statistically significant increases with respect to the characteristic values of the controls $(1.68 \pm 0.12 \mathrm{mPas})$. Magnetic Relaxation is an option to evaluate plasma viscosity because it minimizes the volume of sample needed and eliminates the need to wash the viscometer between determinations. Magnetic Relaxation can compensate its relative high cost, compared with other Viscosimetry methods, facilitating other determinations of utility in several diseases.
\end{abstract}

Keywords: Proton Magnetic Resonance, Dynamic Blood Plasma Viscosity and Hemoglobin

\section{Introduction}

Hyperviscosity syndrome can occur associated with a variety of clinical diagnoses such as polycythemia, Waldenström's macroglobulinemia, multiple myeloma, leukemia, rheumatoid arthritis, asthma, sickle cell anemia and ICTUS [1-3]. This syndrome manifests in the form of bleeding, visual symptoms and neurological deficit that can reach the coma.

The viscosity of the blood plasma and the hemoglobin solution can be determined with capillary, Falling body or rotational viscometers [4] and the method recommended by the International Committee for standardization in hematology is the Harkness viscometer, which is an automated capillary type [5]. However, these viscometers require at least $2 \mathrm{ml}$ of sample, which can reach $10 \mathrm{ml}$ or more, and they need to be washed after each determination, which is an invasive, cumbersome and time-consuming process. On the other hand, not all report the dynamic viscosity, being necessary to determine the density of the sample $(\rho)$ by other methods (pignometry) in order to 
calculate it from the kinematic viscosity $(v): \eta=\rho * v$, which leads to the need for more sample or greater time consumption. Magnetic resonance is, without a doubt, an option to solve these imponderables.

The determination of the dynamic viscosity in blood plasma samples with magnetic resonance methods is based on the patents of the United States of America [6-8].

The aim of the present work is to increase the efficiency and decrease the invasiveness of the current methods for the clinical determination of the viscosity of the blood plasma, from the use of a procedure that decreases by an order of magnitude the amount of sample needed and not it needs the washing of the instrument between measurement and measurement. This procedure allows to evaluate the clinical status and the effectiveness of treatments in diseases where hyperviscosity syndrome may occur.

\section{Materials and Methods}

\subsection{Obtaining Samples of Blood Plasma and Hemoglobin Solution}

The samples of plasma and Hemoglobin solution used to construct the calibration curves, as well as, for the comparison between the methods (control individuals) are obtained through the Provincial Blood Bank "Renato Guitar Rossell", which supplied a frozen bag of $250 \mathrm{ml}$ each time the request for plasma and whole blood was made to obtain the hemoglobin solution.

In the case of patients with Multiple Myeloma and Drepanocytic Anemia, samples of whole blood are obtained in the Clinical Laboratory of the General Hospital "Juan Bruno Zayas Alfonso". The whole blood is obtained by venous puncture and is immediately heparinized. The plasma is obtained by decanting after waiting for the natural process of erythrocyte sedimentation. A set of calibration samples was prepared by continuous dilutions of plasma samples (250 $\mathrm{ml}$ bags) with Saline Buffer Phosphate (PBS), pH 7.4 (SIGMA CHEMICAL Co.).

Obtaining a sample of blood plasma from venous whole blood once the erythrocyte sedimentation time has elapsed, that is, by its natural separation from the rest of the blood content (red blood cells, white blood cells and platelets) and never by the use of other separation methods that cause rupture of the red cells and passage of their cytoplasmic content to the plasma. Between 250 and $500 \mu$ l of plasma will be taken from the top of the liquid column and placed in a tube or tube suitable for Magnetic Resonance studies. The plasma sample will be used immediately to determine the viscosity or stored at room temperature $\left(20^{\circ} \mathrm{C}-25^{\circ} \mathrm{C}\right)$ for up to 4 hours until such determination is made.

The samples of hemoglobin solution was obtained from venous whole blood, these were processed according to the methodology used in the laboratory, which includes extraction of the plasma by aspiration, after extracting the plasma, the red blood cell concentrate was washed three times with phosphate buffered saline (PBS, pH 7.4, SIGMA
Chemicals Co.). Plasma and Buffer after each wash were extracted by aspiration once the sample was centrifuged. Afterwards, hemolysis was carried out by freezing, finally obtaining the desired hemoglobin solution, from which 500 $\mu \mathrm{l}$ samples were prepared in NMR ampoules.

\subsection{Determination of the Viscosity with the Ostwald Viscometer}

The Ostwald capillary viscometer was used as a reference method to determine the viscosity [12]. The Ostwald viscometer, figure 1 , is made of glass. It has a widening in the form of an ampoule provided with two enrases, connected to a vertical capillary tube that joins a second widening intended for the placement of the sample in a first operation, and the water or reference liquid in another complementary operation. The assembly must be kept at a constant temperature so it is usually introduced in a thermostatic bath to set the temperature precisely [13].

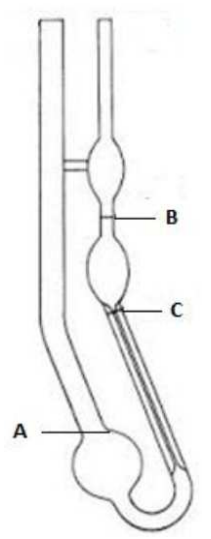

Figure 1. Ostwald viscometer.

The viscometer is filled with the solution to be evaluated up to the upper level, or mark A, then the fluid is suctioned passing the bulb mark through the capillary tube, to the B mark. As a next step, the fluid passes through of the capillary tube under the influence of the hydrostatic pressure thereof. The time it takes to pass the fluid from the B mark to the $\mathrm{C}$ mark through the capillary tube $(\Delta t)$ is recorded. This time is multiplied by a constant of the instrument (coefficient $\mathrm{K}$ ) to determine the kinematic viscosity of the fluid (v) [14]:

$$
v=\mathrm{K} * \Delta \mathrm{t}
$$

Knowing the density of the liquid $(\rho)$ in question, the dynamic viscosity $(\eta)$ is evaluated:

$$
\eta=\rho * v
$$

The value of the coefficient $\mathrm{K}$ of the Ostwald viscometer is reported by the manufacturer or can be determined experimentally.

In the experimental determination of the coefficient $\mathrm{K}$ of the viscometer, $10 \mathrm{ml} \mathrm{[15]} \mathrm{of} \mathrm{distilled} \mathrm{water} \mathrm{are} \mathrm{taken} \mathrm{at} \mathrm{a}$ temperature of $20^{\circ} \mathrm{C}$, the time it takes for the fluid to pass both capillary marks $(\Delta \mathrm{t})$ is determined (10 times), then the 
viscosity and density of the water at the temperature of the experiment are searched in the literature and, using equations 1 and $2, \mathrm{~K}$. is cleared. For the case of our viscometer $\mathrm{K}=$ 1.4402E-7. Knowing the $\mathrm{K}$ of the viscometer and the $\Delta \mathrm{t}$ for a given sample, the kinematic viscosity is calculated [16] (equation 1) but to calculate the dynamic viscosity one must multiply the kinematic viscosity by the density of the liquid at the working temperature (equation 2).

In the Ostwald viscometer, if the experimentally determined $\mathrm{K}$ is used and not the value reported by the manufacturer, the viscosity values obtained by $12 \%$ should be increased.

The dynamic viscosity in blood plasma can be measured in the range between $20^{\circ} \mathrm{C}-37^{\circ} \mathrm{C}$. It can be measured at a temperature and then increase or decrease (depending on the decrease or increase in temperature) by $2.4 \%$ for each degree Celsius of variation up to the desired temperature.

\subsection{Determination of Plasma Density}

For the evaluation of the density a pycnometer (container of exact volume) is used, which is weighed empty and then filled with the plasma, then knowing what a certain volume of liquid weighs density is obtained. It is necessary to determine the volume of the pyknometer if working at a temperature different from that reported by the manufacturer.

\subsection{Operation and Calibration of the MARAN DRX Console}

For the Nuclear Magnetic Resonance measurements, a MARAN DRX console (OXFORD INSTRUMENTS, UK) was used, see figure 2, coupled to a $250 \mathrm{~W}$ RF power amplifier built in our laboratory and a preamplifier (Doty scientific LN-2XL). With this objective, we used the CarrPurcell-Meiboon-Gill pulse series (CPMG) for the evaluation of $\mathrm{T}_{2}$, in a homogeneous magnetic system $\left(\mathrm{B}_{0}=0.095 \mathrm{~T}\right)$. The working temperature (ambient) during the measurements was $20^{\circ} \mathrm{C}$. In our experiments we used glass ampules with $500 \mu \mathrm{L}$ of sample volume. This volume guarantees that the signal-to-noise ratio is adequate to perform the measurements in the equipment, achieving the best homogeneity for the external magnetic field imposed by the system and the radio frequency field that is created in the receiver-transmitter coil [9].

\subsection{Determination of Spin-Spin Relaxation Time $\left(T_{2}\right)$}

For the determination of the spin-spin relaxation time $\left(\mathrm{T}_{2}\right)$, the impulse series Carr-Purcell-Meiboon-Gill (CPMG) was used, which consists of an array of radio frequency pulses that move the magnetization vector $90^{\circ}$ and $180^{\circ}$ [5]. The system is given a pulse of $90^{\circ}$ to obtain a Free Induction Signal (SIL) and then, after a time $\tau$, the sample is given a number $\mathrm{N}$ of pulses of $180^{\circ}$ (separated by $2 \tau$ and out of phase $90^{\circ}$ of the initial pulse) to obtain an echo in the temporal value of $2 \tau$ (corresponding to each pulse of $180^{\circ}$ ).

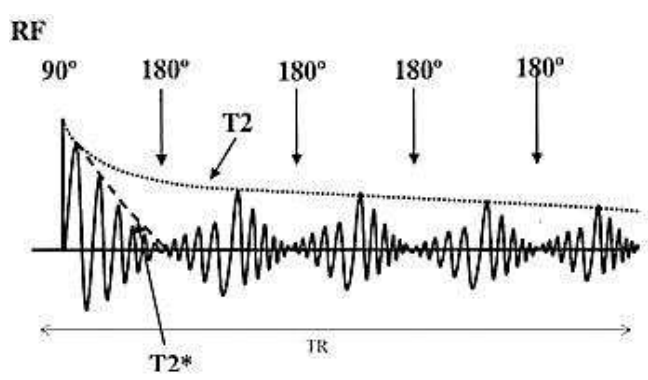

Figure 2. The system is given a $90^{\circ}$ pulse to obtain a Free Induction Signal (SIL) and then, after the time $\tau$, the sample is given a $N$ number of pulses of $180^{\circ}$ to obtain the value temporary 2 de (for each $\tau$ ) an echo.

The fall in the amplitude of the echoes obtained has a biexponential behavior that will be given by the equation:

$$
A(2 m \tau)=A_{0} \exp \left(-\frac{2 m \tau}{T_{2}}\right) \exp \left(-\frac{D G^{2} \gamma^{2} \tau^{2}}{3} 2 m \tau\right)
$$

Where $\mathrm{D}$ is the diffusion coefficient of the molecules that contain the nuclei under study, $G$ is the magnetic field gradient, $\mathrm{A}_{0}$ the initial amplitude of the echoes and $\mathrm{m}$ is an index that runs through the number of echoes from 1 to N. T2 measurement was performed in a homogeneous magnetic system. The determination of Magnetic Resonance will be carried out, at $20^{\circ} \mathrm{C}$, in equipment where the main magnetic field $\left(B_{0}\right)$ is sufficiently homogeneous so that the $G^{2} D$ product is such that the second exponent of the ratio of echo amplitude versus echo time in The recommended series of impulses is equal to unity. The above guarantees the determination of the $T_{2}$ proper to the plasma sample. The $T_{2}$ characteristic of the sample, obtained from the determination by Magnetic Resonance, will be affected by the rapid exchange of water molecules between the linked (a) and free (L) phases of the water in the plasma [10]:

$$
\frac{1}{\mathrm{~T}_{2}}=\frac{\mathrm{P}_{\mathrm{a}}}{\mathrm{T}_{2 \mathrm{a}}}+\frac{\mathrm{P}_{\mathrm{L}}}{\mathrm{T}_{2 \mathrm{~L}}}
$$

Where $\mathrm{Pa}$ and $\mathrm{T}_{2 \mathrm{a}}$ are the fraction and $\mathrm{T}_{2}$ of the water bound respectively and PL and $T_{2 L}$ the fraction and $T_{2}$ of the free water. Considering that plasma is a diluted solution of proteins we can consider that $\mathrm{P}_{\mathrm{L}}>\mathrm{Pa}$ and $\mathrm{T}_{2 \mathrm{~L}}=(2.5-3) \mathrm{s}>$ $\mathrm{T}_{2 \mathrm{a}}[11,1]$. Then equation 2 can be written as:

$$
\frac{1}{\mathrm{~T}_{2}}=\frac{\mathrm{P}_{\mathrm{a}}}{\mathrm{T}_{2 \mathrm{a}}}
$$

\subsection{Statistical Methods Used}

21 samples of control individuals were used to compare the methods developed, as well as 236 of patients with multiple myeloma and 142 of patients with sickle cell anemia to evaluate their medical utility. The normality of all the analyzed data was checked using the statistical program Statgraphics Centurion VI. In all cases it was found that the values of $n$ mentioned were sufficient to ensure that the estimate of the population mean $(\mu)$ was in the interval with $\alpha$ $=0.05$ (95\% reliability) for an error equal to or less than $4 \%$ The same was true for the determinations of $\eta$ in the Ostwald viscometer and in the Magnetic Resonance equipment, in 
which 3 and 10 repetitions were made respectively. The Student's test was used for the comparison between means, with degrees of significance between $\alpha=0.001$ and $\alpha=0.05$. For the comparison of means when the variances are differentiated by at least one order of magnitude, the paired observations test was used [17]. For the analysis of the quality of the linear regression in each of the calibration curves, the determination coefficient $\left(\mathrm{r}^{2}\right)$ was analyzed, this being the square of the correlation coefficient ( $r$ ), an analysis of the residuals [16].

\section{Results and Discussion}

Calibration curve with aqueous plasma solutionsTo determine the dynamic viscosity $(\eta)$ with Nuclear Magnetic Resonance (NMR) methods, it is necessary to find an experimental or theoretical relationship between the NMR parameter that is being determined $\left(T_{2}\right.$ in this work) and $\eta$. A usual procedure is to construct a calibration curve using aqueous solutions at different concentrations as the standard substance. This was implemented by diluting plasma with PBS until having samples with different concentration and different $\eta$, a linear behavior was found $\left(r^{2}=0.99401\right)$, obtaining the equation:

$$
\frac{1}{T_{2}}=0.00185 \eta-0.00102
$$

Where the units of $\mathrm{T} 2$ and $\eta$ are $\mathrm{ms}$ and $\mathrm{mPas}$ respectivelyTable 1 shows the values of $\eta$ in plasma samples and hemoglobin solution of control individuals measured using the Ostwald viscometer and the Nuclear Magnetic Resonance (NMR) method based on the calibration made with plasma samples diluted with PBS (equation 6).

Table 1. Comparison between the values of the viscosity in blood plasma and the Hemoglobin solution with NMR and with the Ostwald viscometer.

\begin{tabular}{lll}
\hline Sample & Viscosity for RMN (cP) & Viscosity with the Ostwald viscometer.(cP) \\
\hline Healthy individuals & $1,51 \pm 0,11$ & $1,45 \pm 0,06$ \\
Healthy individuals & $12,78 \pm 3,55$ & $12,82 \pm 3,35$ \\
\hline
\end{tabular}

Table 1 shows the mean values of the viscosity measured with NMR and the measurement with the Ostwald viscometer, where it can be seen that these values are statistically equal. The student test supports the statistical coincidence between the average dynamic viscosity value determined with the Magnetic Resonance procedure that is presented and that determined with the reference procedure (Ostwald viscometer) for $\alpha=0.10$ (| TT $\mid=$ $0.33267779 \leq \mathrm{t} 0.95=1.47)$. The number of samples used, $\mathrm{n}=$ 20 for blood plasma. This relationship between both methods was supported by the determination of the relationship between the relaxation velocity and the dynamic viscosity made with the calibration curve in plasma.

Table 2. Total patients made by diseases in the case of blood plasma

\begin{tabular}{lll}
\hline & Multiple myeloma & Sicklemia \\
\hline Total patients & 236 & 142 \\
\hline
\end{tabular}

The following table shows the number of patients made by diseases in which the viscosity of the plasma is altered. For multiple myeloma patients, 236 patients were made, these are among debugging, follow-up and hospitalized patients.
Patients suffering from Sicklemia (samples obtained at the Hematology Service of the General Hospital "Juan Bruno Zayas Alfonso" of Santiago de Cuba).

Table 3. Comparison between the mean values of dynamic viscosity of the blood plasma at $20^{\circ} \mathrm{C}$, with the Magnetic Resonance procedure.

\begin{tabular}{ll}
\hline Individuals & Viscosity (cP) \\
\hline Healthy & $1,68 \pm 0,12$ \\
Multiple myeloma & $2,19 \pm 058$ \\
Sicklemia & $2,20 \pm 0,79$ \\
\hline
\end{tabular}

The differences between the mean values of healthy individuals and the different patients can be observed. The dynamic viscosity values in patients with multiple myeloma are significantly higher with respect to healthy individuals, which is explained by the increase that this disease produces in the concentration of plasma proteins. These data demonstrate the usefulness of the procedure developed in the evaluation of the clinical status of the patient with multiple myeloma and the effectiveness of the treatments used.

Table 4. Comparison of the viscosity measured with NMR in Multiple Myeloma for blood plasma.

\begin{tabular}{llll}
\hline & VISCOSITY $(\mathbf{c p})$ & & Toined \\
\cline { 2 - 4 } & Debutante & Tracing & 2,08 \\
MIDDLE VALUE & 2,63 & 0,43 & 1,96 \\
STANDARD DEVIATION & 0,68 & $1,65 \pm 2,51$ & 0,44 \\
RANK & $1,95 \pm 3,31$ & $1,52 \pm 2,40$ & 1,00 \\
\hline
\end{tabular}

Table 4 shows the measured values of the dynamic viscosity by NMR in MM, where it can be seen that the range of the patients debugging patients is between $1.95 \pm 3.31 \mathrm{cP}$, that these results agree with the clinical symptoms and that are patients who are not medicated, and that the normal viscosity is $1.4 \mathrm{cP}$, that of the follow-up patients is between
$1.65 \pm 2.51 \mathrm{cP}$, these results also correspond to the clinic since they are patients that are medicated but relapse due to the disease itself or are complicated by other diseases, admitted patients are between $1.52 \pm 2.40 \mathrm{cP}$, this is due to the fact that patients are complicated with diseases associated with hyperviscosity and that they may present general, 
ophthalmological, neurological, cardiovascular, respiratory, nephrological and urological manifestations, among others.

It is possible to observe the statistically significant differences between the mean values of debugging and treated patients with a $99.9 \%$ reliability. The statistically significant differences between the mean values of healthy individuals and patients treated with $99.9 \%$ reliability can be observed. The values of dynamic viscosity in debutante patients with multiple myeloma are significantly higher with respect to healthy individuals, which is explained by the increase that this disease produces in the concentration of plasma proteins. In the treated patients, a decrease in viscosity is observed as a result of the treatment that does not become statistically the same as in the case of healthy individuals due to the persistence of the disease despite the treatment. This example demonstrates the usefulness of the procedure developed in the diagnosis of the clinical status of the patient with multiple myeloma and in the evaluation of the effectiveness of the treatments used.

Table 5 shows the comparison between the average values of dynamic viscosity determined, at $20^{\circ} \mathrm{C}$, with the Magnetic Resonance procedure, in 30 healthy individuals (samples obtained in the North Hopital Children's laboratory), 42 patients with Multiple Myeloma and 22 patients with sickle cell anemia (samples obtained at the Hematology Service of the General Hospital "Juan Bruno Zayas Alfonso" of Santiago de Cuba).

Table 5. Comparison between the mean values of dynamic viscosity of the hemoglobin solution at $20^{\circ} \mathrm{C}$, with the Magnetic Resonance procedure.

\begin{tabular}{ll}
\hline Individuals & Viscosity (cP) \\
\hline Healthy & $12,78 \pm 3,35$ \\
Multiple myeloma & $13,41 \pm 3,55$ \\
Sicklemia & $12,88 \pm 5,25$ \\
\hline
\end{tabular}

The values of $\eta$ increase in the Myeloma and in Sickle Cell Anemia compared with healthy patients; It is widely known that in Multiple Myeloma this increase is associated with an increase in protein concentration and the same occurs with Sickle Cell Anemia. The protein concentration determines the viscosity of the plasma and the relationship between these two variables is given according to Mooney's law [18].

\section{Conclusions}

1. The viscosity evaluation in Plasma is extremely useful in the clinical evaluation of different diseases.

2. Magnetic Relaxation is an option to evaluate plasma viscosity because it minimizes the volume of sample needed and eliminates the need to wash the viscometer between determinations.

3. Magnetic Relaxation can compensate its relative high cost, compared with other Viscosimetry methods, facilitating other determinations of utility in several diseases.

\section{Acknowledgements}

At the MRI Research Center University of New Brunswick, Canada, for all the support received to complete this work (MRI Research Center of the University of New Brunswick, Fredericton, Canada, for all the support received to finalize this work.). To the Council of Flemish Universities of the Kingdom of Belgium, within the framework of institutional university cooperation program with the Universidad de Oriente. To the workers of the Clinical Laboratory of the Santiago General Hospital for their collaboration in the supply of the blood samples.

\section{References}

[1] Manuel A. Lores Guevara, Yulianela Mengana Torres, Juan C. García Naranjo, Norberto Rodríguez Suárez, Lidia C. Suáres Beyries, María A. Marichal Feliu, Teresa Simón Boada, Inocente C. Rodríguez Reyes, Jan Phillipé. Plasma Dynamic Viscosity Determined by NMR. Applied Magnetic Resonance 2018. DOI 10.1007/s00723-018-1026.

[2] Mette Harreby, et all. Viscosity of Plasma in patients with rheumatoid arthritis. Annals of the rheumatic DiseasesAugust, 1987, 46 (8) 601-604. ISSN: 0003-4967.

[3] Ajayi OI, Famodu AA, Oviasu E. Fibrinogen concentration. A marker of cardiovascular disorders in Nigerians. Turk $J$. Hematol; 2007, 24 (1), 18-22.

[4] Rosencranz, R. and Steven A. Clinical Laboratory Measurement of Serum. Plasma and Blood Viscosity. Clin. Pathol; 2006, 125 (1), 78-86. ISSN: 0974-7532.

[5] Recommendation for a selected method for the measurement of plasma viscosity. Clin. Pathol. 1984, 37 (10), 1147-1152.

[6] A. Shames, Y. Rozenfeld, D. Keinius, Apparatus and method for non-invasive measurement of blood parameters, Patent application number: 2015/0018638A1, Publication date: 15 de Enero del 2015.

[7] P.S. Wellman, M. Blanco, W. Peine, Portable device for $\mathrm{nmr}$ based analysis of rheological changes in liquid samples, Patent application number: US 2015/0130463A1, Publication date: may15, 2015.

[8] Lee Josephson, Rui Hong, Michael J. Cima, Ralph Weissleder, Magnetic resonance-based viscometers and methods, Patent application number: WO 2009/026164A1, Publication date: 26 de Febrero de 2009.

[9] Carlos Cabal Mirabal, Adolfo Fernández García, Manuel Lores Guevara, Evelio González, Leonardo Oramas Díaz. Kinetics studies of complex biomedical process by Magnetic Resonance. Cuban experiences. Applied Magnetic Resonance. https://doi.org/10.1007/s00723-0180985-2

[10] R. S. Menon and P. S. Allen. Solventprotonrelaxation of aqueoussolutions of theserumproteins $\alpha 2$-macroglobulin, fibrinogen and albumin. Biophysical Journal. 1990, 57 (3), 389-396. doi: 10.1016/S0006-3495(90)82555-8.

[11] M. Lores and C. Cabal. Proton Magnetic Relaxation Process during the Polymerization of Hemoglobin S. Appl. Magn.Reson. 2005, 28 (1), 79-84. 
[12] http://scielo.isciii.es/pdf/fh/v39n3/02articuloespecial01.pdf, revisado en marzo 2018.

[13] Sánchez Hernández, B. E. Adolfo López Mateos, Viscosimetro de Bola, Tesis de Licenciatura de Química. Instituto Politécnico Nacional, México. D. F. 2008.

[14] Galego Fernández, N. Manual de prácticas de laboratorio de Química Física de las Superficies y Polímeros. Editorial Pueblo y Educación, La Habana. página 48. 1986.

[15] Lores Guevara, M. A. Estudios preliminares sobre el empleo de los radicales, en seguimiento de la Polimerización de la $\mathrm{HbS}$. Tesis en opción del título de Ingeniero en Física Nuclear. Instituto Superior de Ciencias y tecnología Nuclear. 1993.
[16] Guerra Bustillo C. W., Menéndez Acuña E., Barrera Morera R. y Egaña Morales E. Estadística. Editorial Pueblo y Educación. 1989.

[17] www.quimica.urv.es/quimio/general/callin.pdf, revisado mayo 2018.

[18] Lores Guevara, M. A. 2005. Estudio de los procesos de interacción Magnética y la movilidad molecular durante la polimerización de la $\mathrm{HbS}$ con métodos de Resonancia Magnética. Tesis presentada en opción al título de Doctor en Ciencias Físicas. Universidad de Oriente. Cuba. 